\title{
Koffein verbessert die Motorik
}

Fragestellung: In dieser Studie wurde untersucht, ob eine sechswöchige Behandlung mit Koffein zu einer Verbesserung der Tagesmüdigkeit und der Motorik bei Parkinson-Patienten führt.

Hintergrund: Es gibt Hinweise darauf, dass Menschen, die sehr viel Kaffee trinken, signifikant seltener einen Morbus Parkinson entwickeln. Des Weiteren wissen wir, dass Koffein gegen Tagesmüdigkeit hilfreich ist. Tagesmüdigkeit wiederum ist häufig bei Parkinson-Patienten ein großes Problem, wobei dies sowohl durch das Krankheitsgeschehen im Mittelhirn als auch durch die Medikamente verursacht sein kann.

In jüngster Zeit finden Adenosin-Rezeptor-Antagonisten als Therapeutika zunehmend Interesse im Rahmen der ParkinsonErkrankung. Koffein ist ein nicht selektiver Antagonist des Adenosin-Rezeptors, der wiederum interessante Verknüpfungen zu den motorischen Schleifen im Gehirn aufweist, die bei der Parkinson-Erkrankung defekt sind. In vielen Tierstudien konnte ein positiver motorischer Effekt von Koffein nachgewiesen werden. Dies gelang mittlerweile auch beim Menschen für Adenosin-2A-Antagonisten wie zum Beispiel Preladenant.

Um zu prüfen, ob Koffein auch bei Patienten mit ParkinsonErkrankung zu einer Verbesserung der Tagesmüdigkeit führt und ob Koffein zu einer Verbesserung der Motorik beiträgt, wurde eine sechswöchige randomisierte, placebokontrollierte Doppelblindstudie kreiert.

Patienten und Methode: In dieser Studie wurde über sechs Wochen randomisiert entweder 100-200 mg Koffein zweimal täglich oder Placebo bei Parkinson-Patienten appliziert, die neben der gesicherten Diagnose eines idiopathischen Parkinson-Syndroms auch eine erhebliche Tagesmüdigkeit entsprechend des Epworth-Sleepiness-Scale-Score $(\geq 10)$ aufweisen mussten.

Patienten, die bereits täglich Koffein in einer Dosis von mehr als $200 \mathrm{mg}$ zu sich nahmen, Magengeschwüre hatten, Herzrhythmusstörungen aufwiesen, eine nicht kontrollierbare Hypertension hatten oder andere Gründe für die extensive Tagesmüdigkeit aufwiesen, wurden von der Studie ausgeschlossen. Außerdem wurden Patienten mit Demenz und Depression von der Studie ausgeschlossen.

Die Studie wurde an zwei kanadischen Universitäten und in Brasilien durchgeführt. In den ersten drei Wochen der Studie erhielten die Patienten im Verum-Arm 100 mg Koffein zweimal täglich (beim Aufstehen und nach dem Essen), was danach für weitere drei Wochen in einer Dosierung von zweimal $200 \mathrm{mg}$ täglich fortgesetzt wurde. Die Zeiten der Koffeinaufnahme wurden entsprechend des üblichen Verhaltens, Koffein zum Frühstück und nach dem Mittagessen einzunehmen, angepasst. Ins-

Postuma RB, Rios Romenets S, Zanatta $D$ et al. Caffeine for treatment of Parkinson disease: A randomized controlled trial. Neurology 2012; 79: 651-8 gesamt wurden 61 Patienten in die Studie aufgenommen, von denen 31 im Placebo- und 30 im Koffein-Arm waren.

Ergebnisse: Als primärer Endpunkt wurde die Verbesserung der Epworth-Sleepiness-Scale gewählt, wobei Koffein eine nicht signifikante Verbesserung diesbezüglich erzielte. Im Clinical Global Impression of Change Score fand sich dagegen eine Verbesserung der Somnolenz.

Interessanterweise kam es unter Koffein zu einer signifikanten Verbesserung der UPDRS bis um sechs Punkte im Schnitt nach der sechsten Woche, wobei in der UPDRS Teil III davon drei Punkte erreicht wurden. Lebensqualität, Depression oder Schlafqualität veränderten sich in dieser Studie weder im Placebo- noch im Verum-Arm. Bezüglich der Nebenwirkungen gab es keine signifikanten Unterschiede zwischen den Gruppen.

Schlussfolgerungen: In dieser Pilotstudie konnten ein nennenswerter Koffeineffekt bezüglich der Motorik und eine leichte Verbesserung der Tagesmüdigkeit nachgewiesen werden. Diese überraschend positiven Ergebnisse belegen somit, dass Adenosin-A2-Rezeptor-Antagonisten ein möglicherweise hohes therapeutisches Potenzial haben und dass eine längere und mit mehr Patienten bestückte zweite Studie zur Koffeintherapie bei Parkinson-Patienten gerechtfertigt ist.

\section{-Kommentar von Prof. Heinz Reichmann Koffein im Auge behalten}

Diese Studie eröffnet ein interessantes neues Feld und ist nach den eher enttäuschenden Ergebnissen des ersten AdenosinA2-Antagonisten, Istradefyllin, zusammen mit Daten des neuesten Produkts Preladenant der Beginn für interessante klinische Studien zur Wirksamkeit von Adenosin-RezeptorAntagonisten bei Patienten mit Parkinson-Erkrankung.

Insbesondere dem Koffein sollten wir somit mehr Aufmerksamkeit schenken. Zu prüfen ist, ob diese 61 Patienten uns schon ermutigen sollten, unseren Parkinson-Patienten, die noch keinen Kaffee zu sich nehmen, dies eindringlich zu empfehlen.

Für den Leser mag es interessant sein zu erfahren, dass in einer $150 \mathrm{ml}$ Tasse Bohnenkaffee, die im Filter-Aufguss-Verfahren gebrüht wird, durchschnittlich 80 mg Koffein enthalten sind.

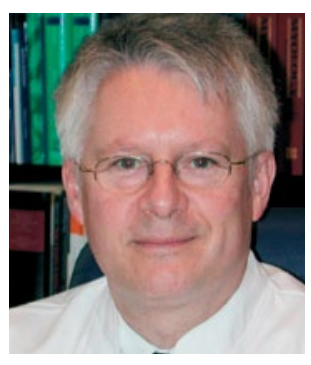

Prof. Dr. med. Heinz Reichmann, Dresden

Direktor der Klinik und Poliklinik für Neurologie, Klinikum Carl Gustav Carus, Technische Universität Dresden E-Mail: Heinz.Reichmann@uniklinikumdresden.de 